\title{
Modelling a complex technical system of greenhouse production: the foundations of an interdisciplinary approach
}

\author{
Evgeniya P. Klyuchka ${ }^{1,}{ }^{,}$, Viktor V. Radin ${ }^{1}$, Leonid M. Groshev ${ }^{1}$, and Valeriy P. Maksimov ${ }^{2}$ \\ ${ }^{1}$ Don State Technical University, 344000 Rostov-on-Don, Russia \\ ${ }^{2}$ Novocherkassk Engineering Institute Reclamation Name A. K. Kortunova, Don State Agrarian \\ University, 346428 Novocherkassk, Russia
}

\begin{abstract}
The fundamentals of an interdisciplinary approach to the design of greenhouse production systems are considered, in which biological objects (plants and humans) are present. The conceptual approach of the software solution is analyzed, synthesizing on the basis of the objectoriented concept such directions as disciplines on the construction of greenhouse production, dynamic simulation models, geoinformation systems. Based on the study of this issue, the conclusion was made about the advisability of applying an interdisciplinary approach for a comprehensive study of the projected complex biotechnical systems of greenhouse production.
\end{abstract}

\section{Introduction}

The statement of the scientific and technical problem of analysis and synthesis of biotechnical systems is determined by the need to develop a general theory of the integration of technical and biological elements in a single control loop for the technological process of greenhouse production. At its core, the synthesis of biological objects and technical systems in greenhouse production has several prerequisites.

The first prerequisite: the emergence of new technologies, innovative technical solutions, software products that are not used and not implemented in greenhouse production. This is due to the complexity of designing poorly formalized multi-component systems of greenhouse complexes, as well as the complexity of introducing new elements, for the purpose of modernization, into already existing technological processes of greenhouse production.

The second premise: a deeper study of the regularities of phenomena in living nature. Despite the fact that they are trying to use the knowledge they have gained about living nature in creating an artificial microclimate in the enclosed space of the greenhouse, the reaction of plants does not give an unambiguous answer to the question: what are the microclimate parameters that are most favorable for maximum productivity. The set of identified factors of different nature (biotic, abiotic) that affect the biological object do not

\footnotetext{
*Corresponding author: klyuchkae@mail.ru
} 
allow us to build a clear methodology for the principles of stimulating action by fields of different nature (electromagnetic, magnetic, electrical, electrochemical, chemical, laser, plasma, optical, etc.).

The third premise: by the middle of the 20th century, on the one hand, certain knowledge was accumulated in the study of the structure of the functioning of living organisms and the basic methods of their study were developed, and on the other, the scientific and technical base necessary for setting up complex multifactor research appeared.

The fourth prerequisite: improvement the methods of mathematical statistics, information theory, the theory of controlled experiment, as well as the technical base development of microelectrode technology, the development of transducer sensors, electronic computers for various purposes, the development of lighting and LED technology.

The fifth prerequisite: the research of leading teams, in a number of countries, carried out in recent years. Creation of artificial intelligence programs; robotic hothouses, without human intervention in the technological process; bionic plant-sensors that react to changes in the environment; development in the field of artificial photosynthesis; with the advent of nanobiotechnology, the introduction of nanotubes into the living structure of plants; plants as an energy source for chargers; development associated with the transfer of electrophysiological information using the computer and microprocessor-based monitor systems, reproduction of living nature in technical systems by the method of functional modeling, based on the requirements of isomorphism of technical systems, formation of databases on research related to the biotechnical system of greenhouse production and the bank of programs for automatic information processing based on the principles of phenotyping and bioinformatics.

The sixth prerequisite: environmental problems associated with greenhouse production, for the solution of which it is necessary to create closed technological processes containing recycles.

The development of scientific methodology in recent years has gone along the path of transition from studying the elements of individual systems to more complex studies of their combinations, connections and interactions. Indeed, at present, fundamentally new tasks have arisen to study the properties of biological objects in order to adequately interface them with technical devices into uniform biotechnical systems. The question of the biotechnical system in greenhouse production, in connection with its novelty, has not yet found wide enough coverage in the literature.

\subsection{Interdisciplinary approach}

The creation of a complex model of greenhouse production is quite difficult from the methodological point of view, since it requires the application of approaches and techniques from various scientific fields. In the most general case, it is about determining the characteristics of individual systems of greenhouse production and its objects. Further, studies are carried out on the efficiency of the selected configuration from several independent systems, taking into account the different elements included in their composition. To perform such an analysis, taking into account all the most influential factors, it is necessary to use a whole range of scientific knowledge from several independent areas, each of which has its own theoretical basis and methodological apparatus. Such areas include:

- modelling natural and climatic conditions in which a greenhouse complex is expected to be operated;

- modelling complex technical systems; 
- design of greenhouse complexes, determination of the structure of greenhouse production and equipment of equipment that ensures the work of the technological process;

- technical calculations of systems and subsystems;

- system analysis, algorithmization and programming;

- modelling of the processes of each separate technological system and, in general, all greenhouse production;

- modelling the functioning of biological objects (plants and humans) in complex technical systems;

- logistic planning of the greenhouse organization;

- technical and economic analysis.

At the same time, specialists of different departmental affiliation are involved in the creation of greenhouse production, and their interpretation of the task of designing the technological process. For example, among the construction professionals of the greenhouse complexes, the solution of the design problem is solved with a remarkable superficial consideration of the factors associated with biological objects and produced at a simple level of the algorithmic study of all aspects of the system. Among the specialists in the automation of technological processes, the priority is the consistency of the operation of a large set of systems (subsystems) to create a microclimate without proper understanding of the biological aspect of the processes occurring. For specialists in the field of biological knowledge, agronomists, artificial microclimate technology, priority is given to the creation of certain agro-technological requirements related to temperature, humidity, light quantity, gas composition, nutrient requirements and water quality. Often the request to an artificial microclimate comes into conflict with the technical capabilities of technological systems that are not able to implement certain environmental conditions of greenhouse plants.

For experts in the field of greenhouse logistics working on modeling the work of greenhouse phytocomplexes, the issues of creating an optimal plan for saving fuel, electricity, water, materials, resources and related tasks are coming to the fore. Simplified models of technological cycles of greenhouse production are used. Different aspects of the specific features of the greenhouse design and the choice of the optimal characteristics of the structure of the greenhouse complex, as a rule, are not taken into account at all, that is, only fixed configurations of the purveyor-fabricator-consumer are considered. For specialists in the financial and economic profile, attention is focused on the economic, managerial and organizational aspects of the creation of a plant-growing greenhouse production with a wide application of regression and statistical estimates of various parameters.

The authors are convinced that in order to improve the quality of greenhouse production, an interdisciplinary approach is required, implementation of which is possible in an integrated software solution, the fundamental principles and architecture of which will provide the opportunity to cover all areas important for the study of such systems. And also research on the creation of a universal software package designed for the design and analysis of greenhouse production systems operating in different climatic conditions. The main idea of this work is to integrate within the framework of a single software methods and approaches from different subject areas on the basis of the following software tools:

- Object-oriented approach;

- Geoinformation environments (GIS);

- Dynamic simulation models. 


\subsection{Object-oriented approach}

The expediency of using the object-oriented approach in research design and the opportunities that are opening up in this connection were formulated back in the 1990s $[1,2,3]$. However, the practical use of the object-oriented approach in applied research, created by construction specialists, is still hampered by the well-known conservatism in the greenhouse sector and the relatively low level of competence in the field of software development. In this case, the paradigm of the object-oriented approach ideally corresponds to the principle of treating the greenhouse as a complexly organized technical system, and the most greenhouse production as a system of a higher hierarchical level in relation to the greenhouse itself as a separate object. A comprehensive work was done on system analysis, decomposition and formalization of the descriptions of the elements of greenhouse production, with a view to further developing the conceptual model and the potential software architecture of the solution being developed. Their full description goes beyond the scope of this article, so let's give an example only of a separate fragment - a description of the structure of the hothouse complex and the technological process.

The logical class "Greenhouse" contains a large number of attributes that describe parameters that do not depend on the state of the system itself. Along with this, its structure contains structures of a complex type ("Groups of microclimate systems" and "Process conditions") in the amount of one or more. As the parameters of these structures, the corresponding characteristics of each individual system and the characteristics of technological processes vary, depending on the degree of its loading (seed preparation, seedling formation, planting in the production zone, the passage of vegetative periods before the ripening of the crop, etc.). The greenhouse can contain an arbitrary number of areas, structural features of the structure, equipment, installations, devices, which are described by a collection of instances of a special class "Equipment Parameters". In this class, it is determined that this "Type of equipment" is used or not used in a given time interval with a given intensity. Temporal segments are divided into seasonal and diurnal, the common characteristics and behavior of which are encapsulated in the abstract class "Time space". Its most important characteristic is the "Description of a specific time", containing the coefficients of time use for each type of technological process, as well as information about which systems in the given time interval are in working order. The logical types of the current state of the greenhouse are divided into two: the general state (how many systems in the working status, at which stage of the process the entire greenhouse, etc.) and the mass state (characterized by the flux density of different types of energy, matter (mass), information).

\subsection{Geoinformation media}

The GIS environment in which the operation of the greenhouse complex is simulated contains data of four types that are stored in different formats $[4,5]$. These data include:

- Layers forming the geographical space in which the greenhouse complex operates, with analysis of relief and ground characteristics;

- layers and databases of natural conditions necessary to calculate the required systems that provide a microclimate with respect to weather conditions, characterized by fluctuations in temperature, humidity, natural insolation, atmospheric pressure, wind load, precipitation, etc.;

- zones with special logical limitations or economic conditions;

- information cartographic layers of weather and climate conditions for seasons for each calendar month separately. 
Description of the geographical space for the operation of the greenhouse complex contains: a temporary space; the current technological cycle (sequential passage of the stages of the cycle); standard algorithms of technological process and maintenance, equipment replacement; a layer of "closed" temporary spaces associated with unforeseen events (equipment failure, plant diseases, etc.). The flow of technological cycles of greenhouse production outside the "closed" temporary spaces is not regulated by predefined algorithms, while within them the movement of the technological process is possible only on fixed predetermined trajectories.

As parameters of a separate technological cycle, such characteristics as sequence and number of current operations will be used; duration of each technological cycle, transitional switching times from one operation to another; composition, the characteristics of the technological cycle, the internal conditions in which the standard technological process must proceed; Observed standard conditions of quality, reliability and safety; probabilistic characteristics of the failure and interruption of the technological process, as well as its stable characteristics - all the parameters that are necessary for calculating the speed of the technological process and the costs of fuel, electricity, water and resources. In this design method, it will be possible to consider existing energy flows (substances) and information flows circulating in the technological space with the analysis of their source, the elements where they are accumulated, in which systems are consumed, and the estimated result of effective use in comparison with the standard proposed process model, and the proposed modified model taking into account the current moment. In general, it can be said that the use of GIS technologies in the modeling of greenhouse production will not only reduce the time required for data preparation, but also provide the possibility of implementing such functions as control, monitoring, and forecasting.

\subsection{Dynamic simulation models}

To analyze the greenhouse production and design of technological processes, stages, cycles, virtualization of various options for comparison and analysis, not only reduces the technological costs, but also provides the opportunity to dynamically develop a complex technical system, modify, restructure, introduce innovative technologies. The composition can use methods

- expert-analytical analysis (definition of the composition of technical systems, their configuration and other parameters of technological production on the basis of analytical formulas and expert estimates);

- The theory of mass service (modeling of work as separate subsystems, their interrelations with other systems, and the description of the operation of the whole system as a whole);

- graph theory and combinatorial optimization (determining the optimal sequence of vegetation stages, technological cycles, scheduling the working phases of the entire production process);

- linear or non-linear mathematical programming (determining the optimal characteristics of products that provide a given cargo flow in the presence of a set of consumers in the system);

- discrete-event or agent simulation (complex analysis and design of interdependent and interrelated technological processes).

Without dwelling on the specifics of these approaches, we note that to compare various greenhouse technologies (hydroponics, aeroponics, gas-discharge and LED lighting systems, single-column, multi-rack, narrow-walled technologies, various lighting systems, etc.), optimize the composition, acquisition of systems and subsystems solution of design tasks) can be used almost all of these methods, taking into account the limitations imposed 
by the organization of work of greenhouse production in a particular climate Based on these considerations, in the structure of the software solution for the study of greenhouse production, computational models of systems (subsystems) serving to determine the composition of technological stages (cycles) and optimize the characteristics of engineering equipment (devices, units, apparatus, installations, engines) imitating agent models for a detailed study of the fixed configuration of the entire complex technical system.

\section{Conclusion}

Not only the complexity of the projected facilities, but also their intensive impact on society and the environment, the severity of the consequences of environmental damage, accidents due to design and operation errors, high requirements to quality and price, and a shortening of the time for the release of new products have become inherent in the modern level of technology development. In modern economic and political conditions, the price of errors in the design of complex technical (technological) systems is very high. The cost of computer simulation studies is much less than the cost of an entire greenhouse infrastructure and the price of possible errors, therefore complex modeling should become a definite standard for research at the modeling, design and operation stages of greenhouse technologies. It is almost impossible to avoid corresponding errors only through the application of expert approaches. For this, an integrated interdisciplinary approach is required, which can be realized in an integrated software solution, the fundamental principles and architecture of which will provide the opportunity to cover all areas important for the study of greenhouse production.

\section{References}

1. V.V. Volkov, A.T. Norov, S.A. Meshkov, Software products and systems, 1, 19 (1996)

2. T. Budd, Object-oriented programming in action: Trans. with English. (Peter, St. Petersburg, 1997)

3. A.L. Fridman, Fundamentals of object-oriented development of software systems (Finance and Statistics, Moscow, 2000)

4. E.V. Pyshkin, Computational, Measuring and Control Systems: Proceedings / Proc. Ed. Yu.B. Senichenkov, 169 (2005)

5. Yu.I. Shokin [and others]; Ed. I.N. Rotanova, Geoinformation technologies and mathematical models for monitoring and management of environmental and socioeconomic systems (Sciences, Sib. Department, Institute of Water and Environmental. Problems, Barnaul, 2011)

6. V.K. Epishin, V.T. Trofimov, Theoretical foundations of engineering geology. Socioeconomic aspects, 32 (1985)

7. A.V. Borshchev, Exponenta PRO, 3-4 (7-8), 38 (2004)

8. A.A. Alexandrov, Vestn. Novosib. state. University. Series: Information technology, 7, 3, 62 (2009)

9. A.E. Radaev, V.A. Leventsov, Theoretical and scientific and practical journal: Organizer of production. The practice of production organization, 3, 30 (2011) 